Document downloaded from:

http://hdl.handle.net/10251/54285

This paper must be cited as:

Castro Palacio, JC.; Velazquez Abad, L.; Gimenez Valentin, MH.; Monsoriu Serra, JA. (2013). Using a mobile phone acceleration sensor in physics experiments on free and damped harmonic oscillations. American Journal of Physics. 81:472-475. doi:10.1119/1.4793438.

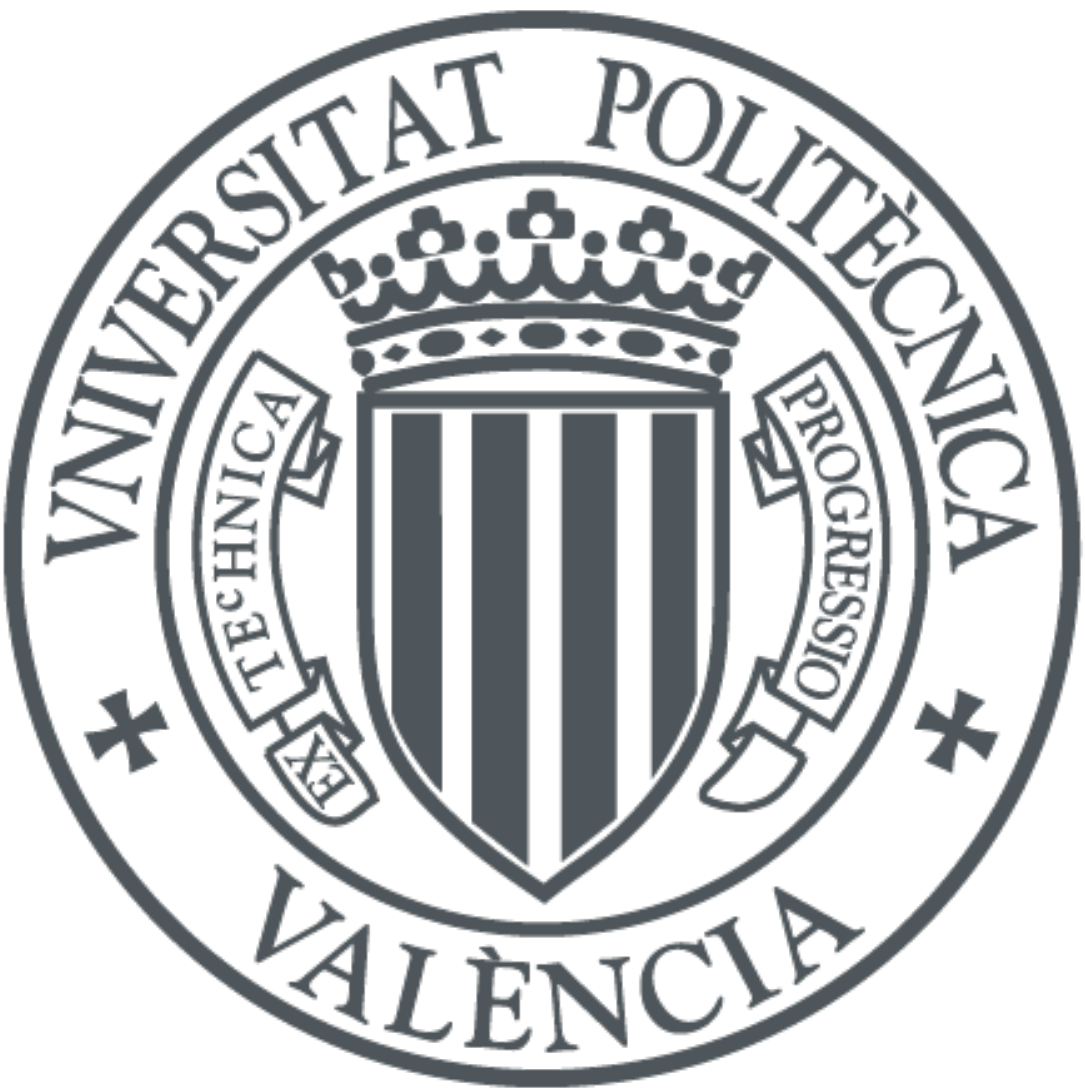

The final publication is available at

http://dx.doi.org/10.1119/1.4793438

Copyright American Association of Physics Teachers

Additional Information 


\title{
Using the mobile phone acceleration sensor in Physics experiments: free and damped harmonic oscillations
}

\author{
Juan Carlos Castro-Palacio \\ Departamento de Física, Universidad de Pinar del Río. Martí 270, \\ Esq. 27 de Noviembre, Pinar del Río, Cuba. CP-20100. \\ Luisberis Velázquez-Abad \\ Departamento de Física, Universidad Católica del \\ Norte. Av. Angamos 0610, Antofagasta, Chile. \\ Marcos H. Giménez \\ Departamento de Física Aplicada, Universitat Politècnica de València, \\ Cami de Vera s/n, 46022, València, Spain. \\ Juan A. Monsoriu* \\ Centro de Tecnologías Físicas, Universitat Politècnica de València, \\ Cami de Vera s/n, 46022, València, Spain.
}

(Dated: November 30, 2012)

\begin{abstract}
The mobile acceleration sensor has been used to in Physics experiments on free and damped oscillations. Results for the period, frequency, spring constant and damping constant match very well to measurements obtained by other methods. The Accelerometer Monitor application for Android has been used to get the outputs of the sensor. Perspectives for the Physics laboratory have also been discussed.
\end{abstract}

*Electronic address: jmonsori@fis.upv.es 


\section{INTRODUCTION}

Electronic portable or every day-use devices offer increasing perspectives for the Physics laboratory at all teaching levels. This is the case of the digital cameras [1], web cams [2], optical mouse of computers [3, 4], XBee transducers [5], wiimote [6] and other game console controllers [7]. For instance, digital techniques have been widely used to visualize Physics concepts [8, 9]. By using a digital camera, a real process can be followed [10, 11]. Then, by analyzing the recorded video a lot of information can be obtained such as distances, time intervals and trajectories of objects. In this way, the study and explanation of complex concepts to the students can be enormously facilitated. More recently, many works related to the use of wireless devices (such as the wiimote) in Physics teaching have been reported $[6,12,13]$. The use of the wiimote for Physics experiments is based on its three axis accelerometer which communicates with the game console using a bluetooth device. Pioneer works about the use of accelerometers in the Physics laboratory can be referred to Weltin [14] and Hunt $[15,16]$. The wiimote includes an infrared image sensor able to follow up to five objects simultaneously, a bluetooth device and three accelerometers.

The wiimote gives Physics teachers a low cost way to track motion in a variety of Physics experiments [17], however, it is not a common device at the Physics laboratories. In this paper, we study the possibility of using another usual accelerometer which almost all students have with them, although they may not realize it. We study free and damped oscillations by using the mobile phone acceleration sensor and the air track. Mechanical oscillations is a very important topic in most of undergraduate Physics courses. Many proposals of Physics laboratory have been published in relation to this topic [18, 19]. For their study, the air track is very useful since the friction force can be appreciably decreased by creating a layer of air between the glider and the track [20]. Even there are many works reporting experiments for the study of oscillations, this is one the first ones that uses a mobile phone as an accelerometer in Physics teaching.

The outline of the paper is the following. In section II, the experimental set up is commented. It includes the introduction to the general features of the free Acceleration Monitor mobile application. In section III, the free harmonic oscillations are studied. This section is divided into two parts: the first for the calculation of the period of the oscillations and the second for the calculation of the spring constant. In section IV, the damping constant 
of the damped oscillations is calculated. Finally, in section V, some conclusions are drawn.

\section{EXPERIMENTAL SET UP}

A photograph of the experimental set up is shown in figure 1. The mobile is placed on the glider of the air track and connected to a fixed end by a spring. Once the air flow goes through the air track the friction is considerably decreased. Under this situation, the glider with the mobile on can oscillate almost freely upon perturbation of the system. The mobile that has been used for the measurements is a Smart phone, LG-E510 bearing an Android version 2.3.4. The mobile mass is $124.0 \mathrm{~g}$ and the air track glider mass is $180.6 \mathrm{~g}$. The mass of the glider can be changed by adding weights at both sides. In this way, the frequency of the free oscillations is changed.

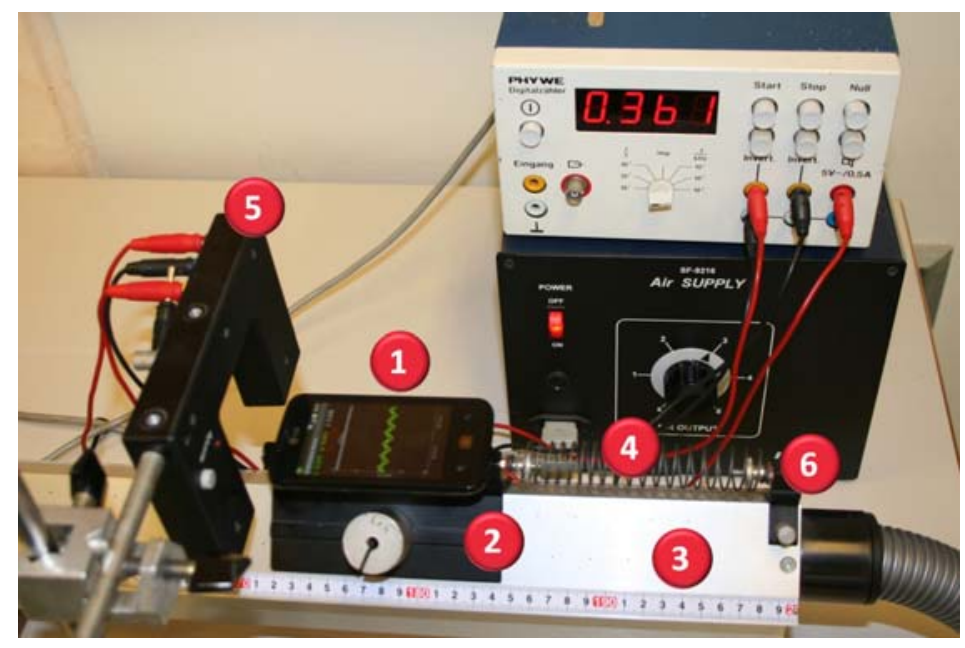

FIG. 1: Photograph of the experimental set up used in the experiments. In the photograph (1) is the smart phone, (2) the glider, (3) the air track, (4) the string, (5) the photometer, and (6) the fixed end.

For the interaction with the mobile sensor, the free Android application "Accelerometer Monitor ver 1.5.0" has been used. This application takes $348 k B$ of SD card memory and it can be downloaded from Google play website at ref. [21]. This application reports the vibrations of the mobile phone in real time by registering the acceleration components on $x, y$ and $z$ - axis at each time step. The component of the acceleration of gravity can be removed from the data. The precision in the measurement of the acceleration is $\delta a=0.01197 \mathrm{~m} / \mathrm{s}^{2}$ 
and for the time is $\delta t=0.02 \mathrm{~s}$. Since the oscillations take place along the $y$-axis, the values of the acceleration for the $x$ and $z$-axis remain very close to zero. This application also allows saving the output data to a file, from which the data can used for further analysis.

Once the application has been downloaded to the mobile device, a small test can be done to check its well functioning. If the mobile is left quiet on a horizontal surface, the application output curves for the acceleration should indicate values very close to zero for all axis.

For the case of damped oscillations, some dissipation of the amplitude of the oscillations can be obtained by decreasing the air flow at the air track.

\section{FREE HARMONIC OSCILLATIONS}

The experimental results of the acceleration $a(t)$ obtained with the Accelerometer Monitor mobile application have been fitted to the following equation:

$$
a(t)=A \sin \left(\omega_{0} t+\phi_{0}\right)
$$

where $A$ is the acceleration amplitude, $\omega_{0}$ is the angular frequency and $\phi_{0}$ the phase constant. The parameters have been obtained by using a least square fitting. In figure 2 , the screen of the Accelerometer Monitor application while displaying free harmonic oscillations has been shown.

In order to start the oscillations in the system, the glider with the mobile was shifted to the right and then left free. The six different masses were obtained by adding weights to both sides of the glider. The output of the mobile application with the acceleration data is collected in an ASCII file. First, a heading with some information such as "saving start date and time", "sensor resolution" and "sensor vendor", can be found. Following the heading, the sensor measurements are shown. The first three columns indicate the acceleration in the three perpendicular axis, $x$ (perpendicular to the device, positive to the right), $y$ (along the device, positive upward) and $z$ (perpendicular to $x$ and $y$ axis, with positive direction as coming out perpendicularly from the device display). In figure 3 , a fragment of the output file is shown.

The scatter of the data points and the fit curve are shown in figure 4 . In table I, the parameters and their errors from the fitting to equation 1 are registered. The quality of the 


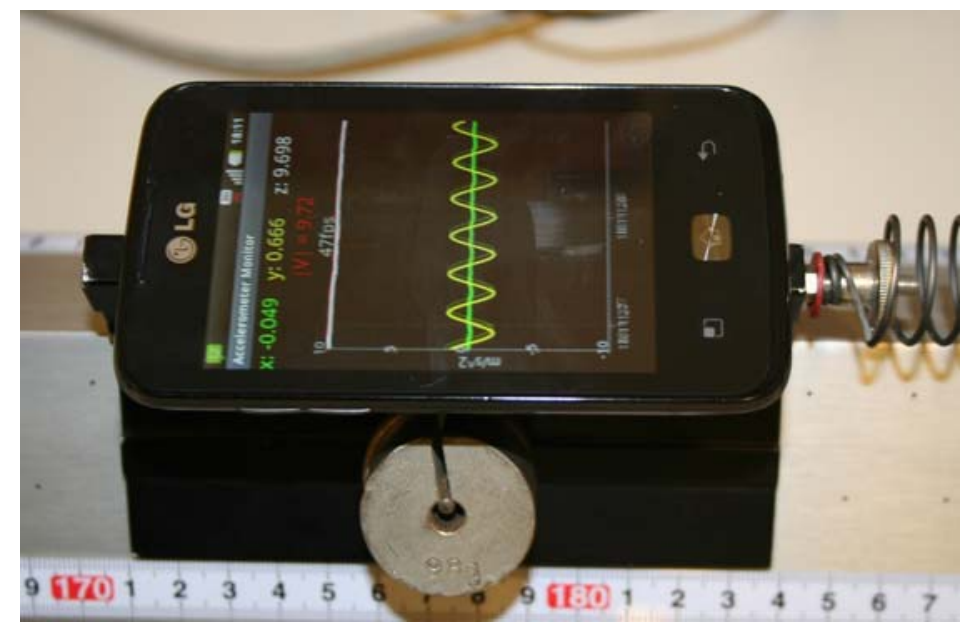

FIG. 2: Photograph of the smart phone displaying a view of the Acceleration Monitor application for the the case of free hammonic oscillations.

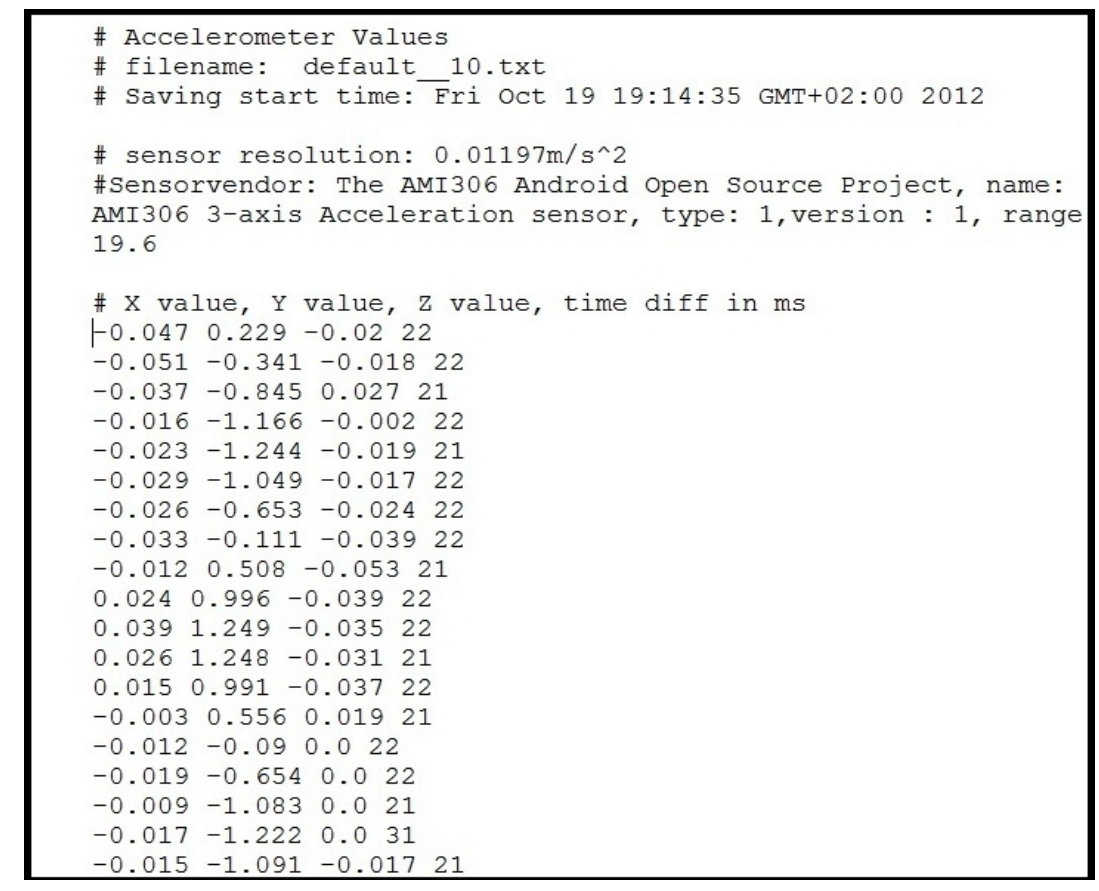

FIG. 3: Fragment of the output data file of the Accelerometer Monitor mobile phone application.

fit can be seen in the values of the regression coefficient $R^{2}$ whose values are close to 1 . 
TABLE I: Parameters and their errors from the fitting of the acceleration data for free oscillations.

\begin{tabular}{ccccccc}
\hline \hline & $(m \pm 0.0001)(\mathrm{kg})$ & $(A \pm \delta A) \mathrm{m} / \mathrm{s}^{2}$ & $\left(\omega_{0} \pm \delta \omega_{0}\right) \mathrm{rad} / \mathrm{s}$ & $\left(\phi_{0} \pm \delta \phi_{0}\right) \mathrm{rad}$ & $R^{2}$ \\
\hline$m_{1}$ & 0.3045 & $1,082 \pm 0,008$ & $24,747 \pm 0,010$ & $-0,797 \pm 0,015$ & 0,9938 \\
$m_{2}$ & 0.4043 & $1,204 \pm 0,006$ & $21,546 \pm 0,005$ & $2,479 \pm 0,009$ & 0,9968 \\
$m_{3}$ & 0.5004 & $1,598 \pm 0,006$ & $19,408 \pm 0,004$ & $-0,377 \pm 0,007$ & 0,9984 \\
$m_{4}$ & 0.6084 & $1,171 \pm 0,007$ & $17,669 \pm 0,006$ & $-0,397 \pm 0,011$ & 0,9953 \\
$m_{5}$ & 0.6285 & $0,856 \pm 0,007$ & $17,371 \pm 0,006$ & $2,553 \pm 0,016$ & 0,9875 \\
$m_{6}$ & 0.6961 & $1,542 \pm 0,008$ & $16,526 \pm 0,007$ & $2,830 \pm 0,011$ & 0,9966 \\
\hline \hline
\end{tabular}

\section{A. Calculation of the period $T$}

In table II, the calculation of the period by using the fitted frequency and the period obtained directed from the photometer are shown. Results indicate a very good agreement between the values since discrepancies are less than $1 \%$ in most of cases.

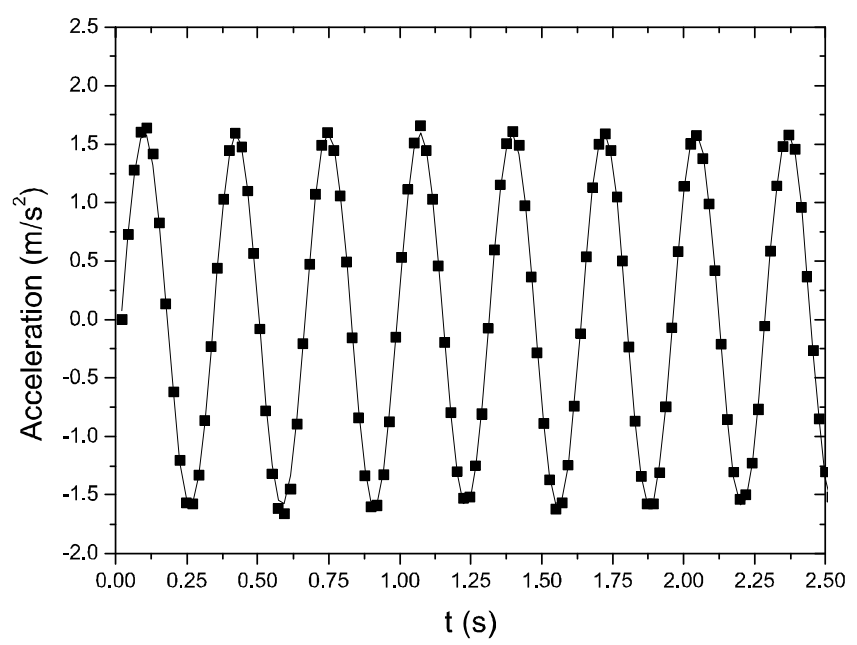

FIG. 4: As an example of the data points (scatter) and fit curves (solid line) of the acceleration versus time, the case of the mass 3 has been shown. 
TABLE II: Periods calculated from the fit (column 2) and the period obtained with the photometer (column 3) are shown. In the fourth column, the percentage discrepancy has been included.

\begin{tabular}{|c|c|c|}
\hline$\left(T_{f i t} \pm \delta T_{f i t}\right) s$ & $\left(T_{\text {Photo }} \pm 0.001\right)$ & $D(\%)$ \\
\hline$m_{1} 0,2539 \pm 0,0001$ & 0,259 & 1,99 \\
\hline$m_{2} 0,2916 \pm 0,0001$ & 0,291 & 0,21 \\
\hline$m_{3} 0,3237 \pm 0,0001$ & 0,323 & 0,23 \\
\hline$m_{4} 0,3556 \pm 0,0001$ & 0,356 & 0,11 \\
\hline$m_{5} 0,3617 \pm 0,0001$ & 0,365 & 0,91 \\
\hline$m_{6} 0,3802 \pm 0,0002$ & 0,380 & 0,05 \\
\hline
\end{tabular}

TABLE III: Data for the calculation of the spring constant.

\begin{tabular}{ccc}
\hline \hline & $\left(\mathrm{m}^{-1} \pm \delta\left(\mathrm{m}^{-1}\right)\right) \mathrm{kg}^{-1}$ & $\left(\omega_{0}^{2} \pm \delta \omega_{0}^{2}\right) \mathrm{rad}^{2} / \mathrm{s}^{2}$ \\
\hline$m_{1}$ & $3,2841 \pm 0,0011$ & $612,4 \pm 0,5$ \\
$m_{2}$ & $2,4734 \pm 0,0006$ & $464,2 \pm 0,2$ \\
$m_{3}$ & $1,9984 \pm 0,0004$ & $376,7 \pm 0,1$ \\
$m_{4}$ & $1,6437 \pm 0,0003$ & $312,2 \pm 0,2$ \\
$m_{5}$ & $1,5911 \pm 0,0003$ & $301,8 \pm 0,2$ \\
$m_{6}$ & $1,4366 \pm 0,0002$ & $273,1 \pm 0,2$ \\
\hline \hline
\end{tabular}

\section{B. Calculation of the spring constant $k$}

Once the values of the mass $(m)$ and the frequency of the free oscillations $\left(\omega_{0}\right)$ have been obtained, the spring constant $k_{f i t}$ can be calculated. For that purpose, a least square linear regression to the equation $\omega_{0}^{2}=k_{f i t} m^{-1}$ has been performed using the values of table III. The string constant has also been calculated by hanging a mass $(m)$ from the spring and measuring the shift in the position $(x)$. For the values, $m=(500,2 \pm 0.1) g$ and $x=(2,6 \pm 0,1) \mathrm{cm}$, we obtained $k=m g / x=(189 \pm 7) \mathrm{N} / \mathrm{m}$. The results for the spring constant $k$ and their errors have been shown in table IV. 
TABLE IV: Results for the spring constant, from the fit (column 1) and using a weight (column 2). In the third column, the percentage discrepancy between both values has been shown.

\begin{tabular}{ccc}
\hline \hline From the fit & \multicolumn{2}{l}{ Using a weight } \\
\hline$\left(k_{f i t} \pm \delta k_{f i t}\right) N / m$ & $(k \pm \delta k) N / m$ & $D(\%)$ \\
$187.9 \pm 0.6$ & $189 \pm 7$ & 0,58 \\
\hline \hline
\end{tabular}

\section{DAMPED HARMONIC OSCILLATIONS}

The damped harmonic oscillations of the acceleration $a(t)$ can be represented by the following equation:

$$
a(t)=D e^{-\gamma t} \sin (\omega t+\phi)
$$

where $D$ is the acceleration amplitude for $t=0 s, \gamma$ is the damping constant and $\phi$, the phase.

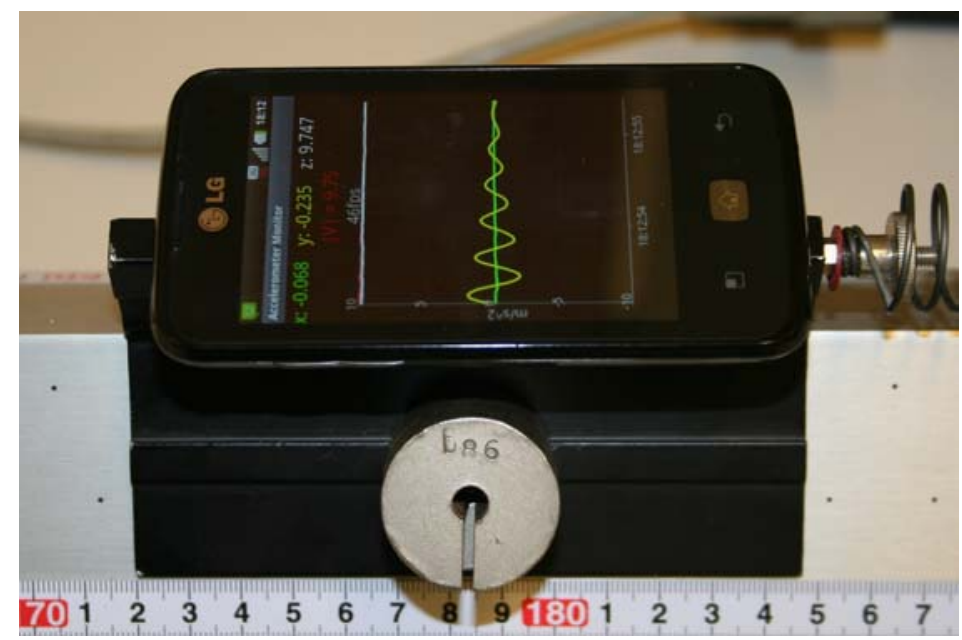

FIG. 5: Photograph of the smart phone displaying a view of the Acceleration Monitor application for the case of damped harmonic oscillations.

In figure 5, the screen of the Accelerometer Monitor application while displaying damped harmonic oscillations has been shown. In figure 6 , the data points and the fit curve are illustrated. In the table $\mathrm{V}$, the parameters and their errors from the fitting to equation 2 
TABLE V: Parameters and their errors from the fitting of the acceleration data for damped oscillations.

\begin{tabular}{lccccc}
\hline \hline & $(D \pm \delta D) \mathrm{m} / \mathrm{s}^{2}$ & $(\gamma \pm \delta \gamma) \mathrm{s}^{-1}$ & $(\omega \pm \delta \omega) \mathrm{rad} / \mathrm{s}$ & $(\phi \pm \delta \phi) \mathrm{rad}$ & $R^{2}$ \\
\hline$m_{6}$ & $1,64 \pm 0,02$ & $0,58 \pm 0,02$ & $16,52 \pm 0,01$ & $-0,56 \pm 0,01$ & 0,9816 \\
\hline \hline
\end{tabular}

using the mass $m_{6}$ are registered. The value of $R^{2}$ which indicates the quality of the fitting has also been included.

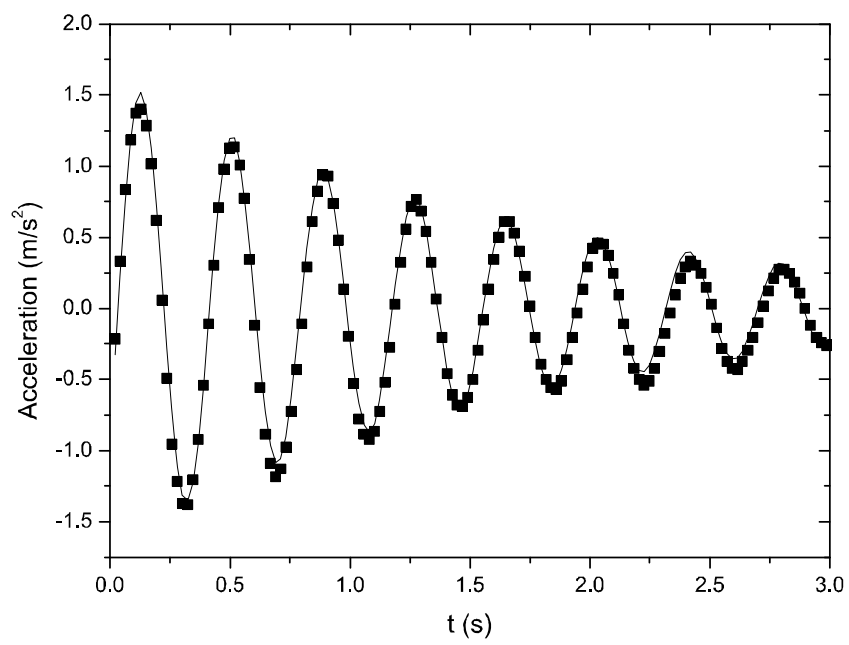

FIG. 6: Data points of the acceleration (scatter) versus time for damped oscillations. The solid line represent the fit curve.

The relaxation time, $\tau$, is the inverse of the damping constant, $\tau=1 / \gamma$. It can also be derived from the following equation:

$$
\tau=\frac{T_{D a} T_{f i t}}{2 \pi} \frac{1}{\left(T_{D a}^{2}-T_{f i t}^{2}\right)^{1 / 2}}
$$

where, $T_{f i t}$, is the period of the free oscillations (see table II) and $T_{D a}=2 \pi / \omega$, the period of the damped oscillations. In table VI, the relaxation times, obtained from the fitting $\left(\tau_{f i t}=1 / \gamma\right)$ and from equation $3\left(\tau_{D a}\right)$, are shown. Results indicate a very good agreement. 
TABLE VI: Results for the relaxation times. The percentage discrepancy between both values has also been included.

\begin{tabular}{crc}
\hline \hline$\left(\tau_{f i t} \pm \delta \tau_{f i t}\right) s^{-1}\left(\tau_{D a} \pm \delta \tau_{D a}\right) s^{-1}$ & $D(\%)$ \\
\hline $1,71 \pm 0.02$ & $1,70 \pm 0,04$ & 0,59 \\
\hline \hline
\end{tabular}

\section{CONCLUSIONS}

Free and damped oscillations have been studied in a very simple way by using the mobile phone acceleration sensor. For this purpose, the free Accelerometer Monitor application for Android operating system have been used. Results for the period, frequency and the spring constant using the mobile sensor data show a very good agreement with the classical way of measuring this quantities in the Physics laboratory. For instance, the period obtained from the fitting is compared to the direct measurement of the photometer and the spring constant, calculated from the fit, is compared to the spring constant calculated from a gravity method using a weight. On the other hand, the damping constant as obtained from the fitting of the mobile phone data allows corroborating theoretical expressions on damped oscillations. This fact offers very good perspectives for the use of the acceleration mobile phone sensor in the General Physics laboratory. For example, the mobile phone as accelerometer sensor can be used to study the most common Physics laboratory pendulums such as the simple, physical and torsion pendulums. It can also be used for the study of two dimensional harmonic oscillations, for example, with the use of the air table [22].

\section{Acknowledgments}

Authors would like to thank the Institute of Education Sciences, Universitat Politècnica de València (Spain), for the support of the Teaching Innovation Group, MoMa.

[1] J. A. Monsoriu, M. H. Giménez, J. Riera and Ana Vidaurre, "Measuring coupled oscillations using an automated video analysis technique based on image recognition", Eur. J. Phys. 26 
(2005) 1149-1155.

[2] S. Shamim, W. Zia, and M. S. Anwar, "Investigating viscous damping using a webcam", Am. J. Phys. 78, 433 (2010).

[3] O. O. Romulo and K. N. Franklin, "The computer mouse as a data acquisition interface: application to harmonic oscillators", Am. J. Phys, 651115 (1997).

[4] T. W. Ng and K. T. Ang, "The optical mouse for harmonic oscillator experimentation", Am. J. Phys. 73793 (2005).

[5] E. Ayars, E. Lai, "Using XBee transducers for wireless data collection", Am. J. Phys. 787 (2010).

[6] S. L. Tomarken, D. R. Simons, R. W. Helms, W. E. Johns, K. E. Schriver et al., "Motion tracking in undergraduate physics laboratories with the Wii remote", Am. J. Phys. 80, 351 (2012).

[7] M. Vannoni and S. Straulino, "Low-cost accelerometers for physics experiments", Eur. J. Phys. 28 (2007) 781-787.cm9tZXRlciJd

[8] A. Vidaurre, J. Riera, M.H. Giménez and J. A. Monsoriu, "Contribution of digital simulation in visualizing physics processes, Comp. App. Eng. Educ. 1045 (2002).

[9] J. Riera, M.H. Giménez, A. Vidaurre and J.A. Monsoriu, "Digital simulation of wave motion", Comp. App. Eng. Educ. 10161 (2002).

[10] H.C. Chung, J. Liang, S. Kushiyama and M. Shinozuka, "Digital image processing for nonlinear systems identification", Int. J. Non-Linear Mech. 39691 (2004).

[11] T. Greczylo and E. Debowska, "Using a digital video camera to examine coupled oscillations", Eur. J. Phys. 23441 (2002).

[12] A. Kawam and M. Kouh, "Wiimote Experiments: 3-D Inclined Plane Problem for Reinforcing the Vector Concept", Phys. Teach. 49, 508 (2011).

[13] R. Ochoa, F. G. Rooney, and W. J. Somers, "Using the Wiimote in Introductory Physics Experiments", Phys. Teach. 49, (2011).

[14] H. Weltin, "Accelerometer", Am. J. Phys. 34, 825 (1966).

[15] J. L. Hunt, "Forced and damped harmonic oscillator experiment using an accelerometer", Am. J. Phys. 53, 278 (1985).

[16] J. L. Hunt, "Accurate experiment for measuring the ratio of specific heats of gases using an accelerometer", Am. J. Phys. 53, 696 (1985). 
[17] A. Skeffington and K. Scully, "Simultaneous tracking of multiple points using a wiimote", Phys. Teach. 50, 482 (2012).

[18] P. Onorato, D. Mascoli, and A. De Ambrosis, "Damped oscillations and equilibrium in a mass-spring system subject to sliding friction forces: Integrating experimental and theoretical analyses", Am. J. Phys. 78, 1120 (2010).

[19] G Flores-Hidalgo and F A Barone, "The one-dimensional damped forced harmonic oscillator revisited", Eur. J. Phys. 32 (2011) 377-379.

[20] J. Berger, "On potential energy, its force field and their measurement along an air track", Eur. J. Phys. 947 (1988).

[21] https://play.google.com/store/apps

[22] N. C. Bobillo-Ares and Jose Fernandez-Nufiez, "Two-dimensional harmonic oscillator on an air table", Eur. J. Phys. 16223 (1995). 